\title{
IL POTERE DEL LINGUAGgIO E LE NARRATIVE PROCESSUALI
}

\section{Alberto Vespaziani ${ }^{1}$}

\begin{abstract}
RIASSUNTO: il testo indaga la relazione ambivalente che lega il potere del linguaggio al linguaggio del potere; quindi esplora il concetto di narrazione, nella doppia dimensione di narrazione nel processo e di narrazione come processo. La narratività viene discussa sia in riferimento alla giurisprudenza costituzionale, sia alla costituzione stessa, intesa come un processo pubblico. Lo studio culturale del diritto considera il linguaggio giuridico non come uno strumento, ma come un insieme di segni che richiedono interpretazioni plurali.
\end{abstract}

Parole-Chiave: linguaggio; potere; narrazione; costituzione; giurisprudenza; processo.

La relazione tra linguaggio e potere è una relazione ambigua. Osservata dal lato del diritto, essa sembra focalizzarsi sul potere: il diritto è il linguaggio del potere, quindi il linguaggio non è che uno strumento che il diritto mette al servizio del potere. Osservata dal lato della letteratura, viceversa, essa sembra incentrarsi sul linguaggio: attraverso il corretto uso della lingua e dei concetti giuridici il potere può essere addomesticato, reso controllabile e, quindi, giusto. Per una prospettiva di "diritto e letteratura" proprio questa ambiguità si rivela essere estremamente produttiva ed insita nello stesso fenomeno giuridico. Essendo un giurista, non tratterò il problema dal lato della teoria letteraria o della filosofia del linguaggio, ma piuttosto dal lato dei fenomeni giuridici, in particolare dell'intreccio tra giuridicità e narratività. Il contributo si divide in tre parti: nella prima parte svolgerò alcune brevi considerazioni sulla relazione ambivalente che lega il potere del linguaggio al linguaggio del potere; nella seconda mi concentrerò

\footnotetext{
1 Dottore in Diritto Costituzionale presso l'Università La Sapienza di Roma (Italia). Professore Associato di Diritto Pubblico Comparato nell'Università degli Studi del Molise (Italia). E-mail: alberto.vespaziani@unimol.it
} 
sul concetto di narrazione, nella doppia dimensione di narrazione nel processo e di narrazione come processo; nella terza parte svilupperò alcune riflessioni generali sugli aspetti culturali e pluralistici che contraddistinguono gli studi di diritto e letteratura oggi.

\section{IL POTERE DEL LINGUAGGIO}

\section{E IL LINGUAGGIO DEL POTERE}

Mediante il linguaggio noi comunichiamo, ci organizziamo, regoliamo i nostri comportamenti, ma nello stesso tempo nell'uso dei nostri linguaggi noi produciamo dei fraintendimenti, diffondiamo disinformazione e introduciamo confusione nei rapporti sociali. Quello che dal punto di vista della letteratura può sembrare uno strumento meraviglioso per la creazione di storie edificanti, dal punto di vista del diritto, in particolare del diritto costituzionale, appare essere tanto uno strumento di disciplina e controllo del potere, quanto uno strumento a servizio delle tendenze distruttrici del potere.

Dal punto di vista della letteratura l'ambiguità del linguaggio è costitutiva della pluralità di senso; come ha notato Roland Barthes (2002, p. 46) "se le parole avessero un senso solo, quello del dizionario, se una seconda lingua non venisse a sconvolgere e liberare "le certezze del linguaggio", non ci sarebbe infatti letteratura”.

Dal punto di vista del diritto assume particolare importanza il discorso sui limiti del linguaggio, e quindi, sui limiti del potere. La rappresentazione dominante del costituzionalismo tende a concepirlo come un insieme di tecniche e discorsi volti al controllo e alla limitazione del potere: il linguaggio costituzionale viene presentato come uno strumento al servizio del fine (buono) del mantenimento dell'ordine politico e delle libertà individuali e sociali. Ma ogni riflessione sul linguaggio, e in particolare sul linguaggio giuridico, incontra presto la dimensione del limite, inteso sia come argine al potere, una zona di rispetto dei diritti fondamentali, sia come incapacità del linguaggio giuridico di tutelare $i$ valori fondamentali e le storie fondative delle comunità. Nel capolavoro "Bartleby lo scrivano" Melville ha indagato i limiti del paradigma giuridico e gli scogli insormontabili che il linguaggio giuridico incontra di fronte al misterioso silenzio e all'ostinato rifiuto di Bartleby che si limita a balbettare 
"I prefer not to". I dialoghi tra l'avvocato e Bartleby illustrano i limiti del paradigma giuridico: quanto più l'avvocato cerca di comprendere le ragioni di Bartleby, tanto più questo si ritira nel suo rifiuto immotivato: quanto più l'avvocato intensifica la giuridicità delle sue argomentazioni, tanto più lo scrivano si ritrae nel suo silenzio incomprensibile. Tuttavia se la comunicazione giuridica dell'avvocato non riesce a salvare Bartleby dal suo silenzio autodistruttivo, il suo racconto riesce a restituire valore e memoria ad un'epoca scomparsa del diritto e ad un'esistenza perduta (Vespaziani, 2012, p. 80).

Come il costituzionalismo costruisce e difende i limiti del potere politico, la letteratura costruisce un insieme di storie e di racconti che tuttavia riescono solo a lambire ciò che si può dire e rappresentare: il silenzio, tanto del diritto quanto della letteratura, costituiscono non solo i confini del dicibile, ma anche una modalità, forse la più profonda, della comunicazione umana. Lo sforzo di chiarificazione del ragionamento giuridico e il tentativo di affabulazione del racconto letterario incontrano un comune limite nel silenzio, nella impossibilità di comprendere fino in fondo il proprio mondo, la propria epoca e i linguaggi che la costruiscono e costituiscono.

Ecco dunque profilarsi una nuova ed insidiosa ambiguità: da una parte la creazione di storie contribuisce al consolidamento di comunità. La diffusione di narrazioni di movimenti politici, di gesta eroiche, di avvenimenti istituzionali, ma anche di storie letterarie condivise, di architetture e musiche comuni, di opere d'arte rappresentative, tutti questi fenomeni culturali contribuiscono alla fondazione e al consolidamento di comunità sociali e politiche.

Le società umane non vivono di solo diritto o di lotte per il potere, esse si tengono insieme principalmente grazie alle opere culturali che forniscono il medium simbolico che permette la solidarietà tra estranei. Come italiano, non posso ricordare che l'unificazione culturale italiana è avvenuta almeno a partire dalla diffusione delle opere letterarie di Dante, Petrarca e Boccaccio, dunque nei secoli XIII e XIV, mentre l'unificazione politica è avvenuta (meglio, cominciata) nel 1861 !

D'altra parte la crisi del paradigma narratologico, la difficoltà delle società contemporanee di riconoscersi in storie condivise, mostra anche 
come la crisi delle narrazioni comuni contribuisce ai dolorosi fenomeni di disgregazione di comunità. La nostra epoca postmoderna mostra come lo sviluppo di crisi politiche e costituzionali sono spesso accompagnate da crisi del senso di appartenenza alla stessa comunità politica, allo stesso spazio pubblico condiviso. Il trionfo del paradigma comunicativo è l'altra faccia della medaglia della crisi della soggettività moderna, che aveva accompagnato il consolidamento degli ideali democratici nel XX secolo.

Ecco allora che la diffusione del movimento "diritto e letteratura" costituisce un fenomeno tipico della nostra epoca; più che un'esigenza di raffinatezza culturale o di abilità interpretativa, esso segnala la profonda inquietudine dei nostri tempi e rappresenta l'esigenza di ricostituire dei linguaggi condivisi e delle narrazioni significative, capaci di orientare il senso dei giuristi e dei letterati nelle nostre società interconnesse, ma liquefatte.

Come aveva notato sapientemente Roland Barthes (1988, p. 95), l'etimologia della parola "narrazione" è da rintracciare nel latino "gnarus", colui che conosce in una maniera particolare. Narrare è dunque l'attività, tanto letteraria quanto giuridica, che indica i processi mediante cui trasformare il sapere in dire. La letteratura è certo un campo in cui alcune conoscenze vengono trasformate in narrazioni, ma non vi è dubbio che un luogo privilegiato in cui i fatti avvenuti vengono rappresentati con lo scopo di pervenire a decisioni vincolanti è il processo giurisdizionale. Passo quindi a discutere i due sensi principali dell'intreccio tra narrazione e processo.

\section{LE NARRAZIONI NEL PROCESSO E COME PROCESSO}

"To the extent that law is fact-contingent, it is inescapably rooted in narrative" (Amsterdam e Bruner, 2000, p. 111). In un primo senso più ristretto le narrazioni avvengono all'interno dei processi; nelle aule dei tribunali i testimoni raccontano la loro versione dei fatti, i periti certificano le modalità in cui alcune azioni si sono svolte, gli avvocati tentano di persuadere giurie o giudici togati costruendo trame narrative, $\mathrm{i}$ giudici argomentano nelle loro decisioni le ragioni che le hanno condotte a preferire una narrazione rispetto ad un'altra. 
Ad una prima approssimazione possiamo insistere su due distinzioni: la differenza tra racconto dei fatti e narrazione delle regole, nonché la distinzione tra narrazione e argomentazione. Cominciamo dalla prima.

Anzitutto i processi che si svolgono nei tribunali debbono accertare che cosa è successo, e per far ciò debbono affidarsi a ri-costruzioni narrative della realtà. L'educazione giuridica classica dà troppa importanza alla conoscenza delle regole positive e sottovaluta la dimensione letteraria della ricostruzione dei fatti. Nelle aule di giustizia i fatti non hanno una consistenza ontologica incontrastata, essi sono al centro di dispute, di disaccordi, di argomentazioni e di prove. Solo grazie alla logica procedurale della prova si può "tenere per vero" un fatto, lo si può "dare per avvenuto".

A ben vedere, solo l'esigenza economica di impedire un giudizio infinito richiede che ad un certo punto si finisca di produrre argomenti e giudizi, e si accetti come effettivamente avvenuto ciò che è contenuto nel "passaggio in giudicato". Ecco allora che nell'argomentazione giuridica assume grande importanza il modo in cui le parti raccontano lo svolgimento degli avvenimenti. Nel suo magistrale libro Poetic Justice, Martha Nussbaum (1995, p. 99) ha mostrato esempi illuminanti sulle strategie narrative di presentazione dei fatti, e come queste hanno una grande rilevanza nelle scelte di valore che sottendono le decisioni sulle norme da applicare. Non a caso, proprio sull'intersezione tra "diritto come letteratura" e teorie della prova sono fiorite le teorie narrativiste del diritto (Di Donato, 2008, p. 79).

D'altro canto anche la costruzione argomentativa delle norme positive rilevanti, degli argomenti accettabili e dei precedenti vincolanti (nella common law) mostra una rilevanza narratologica. Proprio rispetto al modello argomentativo del giudice della common law, Ronald Dworkin (1986, p. 228) propose il paragone con la chain novel: come i singoli capitoli di un romanzo pubblicato a puntate si legano in una catena narrativa, così le decisioni dei giudici che rinviano a precedenti e alla costituzione costituiscono un adeguamento e una giustificazione ( $f$ it and justification) della validità del procedimento argomentativo, nonché delle norme e delle decisioni presupposte. 
Indubbiamente la giurisprudenza è il luogo ideale per osservare la natura narrativa dell'argomentazione. Incidentalmente osserviamo una differenza strutturale tra la natura del linguaggio del diritto legislativo e quella del diritto giurisprudenziale: mentre il primo mira alla prevedibilità, si dirige alla generalità ed al controllo sociale futuro, il secondo articola una soluzione per un caso particolare, si indirizza all'individualità ed alla composizione di un conflitto passato. Ogni lettore può constatare la differenza tra la lettura della Gazzetta Ufficiale e di una rassegna di giurisprudenza. Il linguaggio del legislatore è astratto, analitico e formale; il linguaggio del giudice è concreto, dialettico e narrativo. Il legislatore deve soddisfare gli interessi della maggioranza che ha vinto le elezioni, egli assiomaticamente dispone; il giudice, invece, deve persuadere la parte soccombente ed argomentare le ragioni giuridiche che motivano la sua decisione.

I racconti giudiziari hanno struttura narrativa, spirito antagonistico, finalità intrinsecamente retorica e sono giustificabilmente esposti al sospetto. Sono modellati su casi precedenti i cui verdetti sono ad essi favorevoli. E infine, mirano seriamente ai risultati, giacché le parti in causa debbono avere legittimazione attiva e un interesse diretto per l'esito finale. Narrativi, antagonistici, retorici e partigiani! (Bruner, 2002, p. 49)

La natura narrativa dell'argomentazione giudiziale si evidenzia soprattutto nella giurisprudenza sui diritti fondamentali: un caso che coinvolge la violazione di un diritto fondamentale presenta l'accadere di un evento inatteso, il cui racconto comincia con qualche infrazione dell'ordine prevedibile delle cose: "la narrativa in tutte le sue forme è una dialettica fra ciò che si attendeva e ciò che è stato. Perché vi sia un racconto, occorre che accada qualcosa di imprevisto, altrimenti non c'è storia" (Bruner, 2002, p. 17).

La giurisprudenza costituzionale sui diritti fondamentali somiglia molto alla peripéteia aristotelica; essa presenta un evento inatteso, imprevisto dal legislatore eppure accaduto nella realtà. La giurisprudenza costituzionale contemporanea ci presenta una serie di racconti di eventi inattesi che cominciano con un'infrazione dell'ordine delle norme predisposte dal legislatore, un'insoddisfazione e una ribellione nei confronti del diritto legislativo mosse dalla rivendicazione di un diritto fondamentale. 
Da questo punto di vista la struttura narrativa della giurisprudenza costituzionale sui diritti fondamentali è simile alla struttura della tragedia greca: in una situazione iniziale di ordine stabilito si verifica un evento perturbatore che sconvolge i rapporti esistenti; solo la catarsi della decisione può ristabilire l'ordine iniziale, oramai trasfigurato in una nuova condizione di equilibrio. Sia il giudice costituzionale, sia il coro della letteratura tragica non si limitano a descrivere l'esistente e a prescrivere il doveroso mediante un linguaggio indicativo; essi mostrano anche ciò che potrebbe essere, che sarebbe potuto essere, spingendosi nella dimensione tipicamente narrativa del congiuntivo e del condizionale.

La grande giurisprudenza costituzionale (les grands arrêts e i leading cases), come "la grande narrativa, è, in spirito, sovversiva, non pedagogica. [...] Dopotutto la sua missione è ridare stranezza al familiare, trasformare l'indicativo in congiuntivo" (Bruner, 2002, p. 13). La congiuntivizzazione consiste proprio nel mostrare ciò che potrebbe essere, la narratività come il ponte tra ciò che è e ciò che deve essere. La lotta per i diritti fondamentali presenta dunque un mismatch, una mancata corrispondenza tra l'essere e il dover essere che può essere colmata solo presentando un discorso nuovo rispetto all'ordine costituito dello stato di fatto. Mentre Dworkin (1985, p. 158) vedeva nella chain novel della giurisprudenza costituzionale una continuità del fit and justifies, una catena narrativa conseguenziale in cui la decisione si inserisce, Bruner individua nel lack of fit la natura profonda del caso che richiede una nuova pronuncia, una estraniazione del familiare ed una familiarizzazione dell'estraneo.

Ecco che mentre la legislazione costituzionale assolve alla funzione di limitare il potere, disciplinandone l'organizzazione, la giurisprudenza costituzionale permette il perseguimento di nuove possibilità e la soddisfazione di interessi individuali fondamentali.

Ma tra la narratività letteraria e quella giuridica sussistono importanti differenze:

un buon racconto e un'argomentazione ben costruita rappresentano due generi di cose ovviamente molto diversi tra loro. È vero che ci si può servire di entrambi per convincere un'altra persona; ma le cose di cui essi convincono sono fondamentalmente diverse tra loro: le argomentazioni ci convincono della propria verità, i racconti della propria verosimiglianza. Le une sono suscettibili di verifica, appellandosi in definitiva alle 
procedure con cui si elabora una dimostrazione formale o empirica; gli altri non stabiliscono la verità, ma la verosimiglianza (Bruner, 2005, p. 15).

Tra le narrazioni letterarie e quelle giudiziarie corre la stessa differenza che c'è tra verità e verosimiglianza: mentre le arti narrative e persuasive si concentrano sulle emozioni, sul verificarsi di un evento imprevisto che rompe un ordine prestabilito per ristabilirne uno nuovo, le arti giuridiche e argomentative si fondano sul controllo razionale delle ragioni addotte (topica), delle storie raccontate, e ne chiedono l'inserimento in una struttura logica che si conformi ad ideali di coerenza.

In questo senso alcuni stati di tradizione giuridica di civil law (Francia, Italia, Belgio...) hanno sviluppato un vero e proprio apparato istituzionale deputato al controllo giuridico-formale delle argomentazioni dei giudici: le Corti di Cassazione. Per fortuna non esiste nessuna Cassazione per la letteratura! Tra la narratività letteraria e l'argomentazione giuridica sussiste insomma un'importante differenza quantitativa riguardo la libertà creativa: lo scrittore di racconti, ma anche il critico letterario, godono di una libertà affabulatoria ed interpretativa assai maggiore di quella del legislatore $\mathrm{o}$ del giudice. La letteratura può essere più inverosimile o fantastica di una legge o di una sentenza. Il romanzo può nutrirsi di sola verosimiglianza o finanche di sola falsificazione, una decisione giudiziaria deve quantomeno tentare la maggiore approssimazione possibile alla realtà.

Nell'indagare le narrazioni processuali mi sono sinora concentrato sulla costruzione di storie e di narrative all'interno del processo giudiziale, ma vi è anche una dimensione più ampia in cui si situa l'intreccio tra giuridicità e narratività, quella della 'costituzione come un racconto'. Questa formulazione, tanto suggestiva quanto fuorviante, veicola a mio avviso due concezioni da cui intendo prendere la distanze: la costituzione come prodotto di una reificazione, e l'autoreferenzialità postmoderna del genere narrativo.

Cominciamo con la prima concezione: la parola "costituzione" si connota per la sua altissima polisemicità; in nessuna dottrina giuridica vi è accordo su di un unico concetto di costituzione, anzi spesso le voci enciclopediche che hanno per oggetto il lemma "costituzione" si risolvono 
in dei minitrattati di storia delle dottrine politico-costituzionali. Ma oltre il disaccordo interpretativo sulla portata e sul significato della parola "costituzione" le lingue romanze, mediante l'articolo determinativo, tendono a reificare le costruzioni concettuali. La riflessione del giurista costantemente riduce e traduce l'esperienza in un mondo di parole e la verbalizzazione romanza dell'esperienza costituzionalistica inevitabilmente rafforza il pregiudizio ontologico.

Come ci ricorda Riccardo Orestano,

l'abbandono dell'impiego della lingua latina da parte della giurisprudenza ha ancor più incrementato l'uso delle astrazioni. Le lingue moderne infatti - come già la lingua greca - hanno la possibilità di parlare per astratti in misura assai più ampia di quanto non consenta il latino. In esse la presenza dell'articolo determinativo rende agevole, proprio come fatto linguistico, l'assegnare valore astratto a nomi concreti (ponendoli come "oggetti" universali), l'attribuire parvenza di concreto a nomi astratti (presentandoli come "oggetti" universali), il sostantivare forme verbali e aggettivi (dando una determinazione all'immateriale e introducendoli nel discorso quali "oggetto di pensiero", su cui diviene possibile formulare giudizi come su una "cosa"). L'articolo determinativo stimola così la formazione di concetti astratti, offrendo al pensiero una serie infinita di "oggetti" che vengono elevati a nozioni universali, attraverso il mezzo linguistico e l'elemento logico implicito nelle categorie grammaticali. [...] Fatto è che da quando i nostri giuristi hanno potuto impiegare espressioni come "il diritto soggettivo", "la obbligazione", "il contratto", "il diritto reale", "la azione" e via dicendo, il mondo del diritto si è popolato di ipostasi e di edificazioni ancor più numerose e ancor più impegnative sul piano dell'astrazione di quante ne avessero create due millenni di riflessione di speculazione giuridica, con le sue costruzioni in genera e species, con le sue definitiones e con la ricerca della loro "essenza", nonché con i suoi procedimenti di riduzione dell'esperienza a ontologia. Diversamente gli inglesi che hanno sempre parlato di contract, di action ecc. senza premettervi alcun articolo (Orestano, 1987, p. 393).

Parlare del"la" costituzione, secondo questa prospettiva, implica una reificazione di un processo, un'astrazione concettuale di un'esperienza che viene convertita in un oggetto del pensiero, bisognoso di definizione e classificazione. Per un approccio "diritto e letteratura" viceversa, la costituzione non è l'oggetto di un racconto, ma

il raccontare nel suo svolgimento, il suo dispiegarsi nel tempo: il suo articolarsi e distribuirsi in collegate partizioni, il suo progressivo, ritmico, distendersi o 
concentrarsi; un soggetto che si racconta e che racconta. Un processo corale, nel quale risultano coinvolti, ciascuno a modo suo, ed al suo posto - come vorremmo nelle contemporanee esperienze del pluralismo - una molteplicità di attori e, anzi, di autori: innumerevoli persone chiamate - nel consenso, ma anche nel dissenso, individuale o collettivo - ad aggiungere (auctor da augeo) qualcosa di proprio, ad accrescere, a far crescere un patrimonio comune di eticità e di razionalità divenuto come un'immensa e straordinaria enciclopedia. Un albero che, secondo una vecchia immagine del sapere, si ramifica, moltiplicando le strutture del fusto ed espandendo la linfa. Autori e "decisori", e tuttavia, allo stesso tempo, come si dice, interpreti: artefici, infatti, di opinioni, di originali ri-proposizioni; non semplicemente di testi, ma di contesti. In processi costituenti destinati a risultare inevitabilmente continui (De Nitto, 2005, p. XXIV).

Il diritto costituzionale dunque, più che essere "il" diritto del"la" costituzione, esprime una modalità narrativa di esperienze condivise. Esso non è neanche "il" racconto del"la" costituzione, un discorso narrativo tra i tanti, un altro genere letterario. Il diritto costituzionale è piuttosto un insieme di precetti normativi e di racconti significativi che orientano il senso di marcia e di conflitto di una comunità. Se il racconto costituzionale fosse soltanto un genere letterario, non si spiegherebbero né la violenza legittima dell'apparato statale, né le lotte per il riconoscimento delle comunità che pretendono la tutela pubblica dei propri diritti.

Tzvetan Todorov ha scritto pagine memorabili sul pericolo corso dalla rappresentazione post-moderna dell'autoreferenzialità letteraria, l’idea seconda la quale dietro le storie ci sono solo altre storie e quindi lo studio della letteratura è fine a se stesso:

la letteratura può molto. Può tenderci la mano quando siamo profondamente depressi, condurci verso gli esseri umani che ci circondano, farci comprendere meglio il mondo e aiutarci a vivere. Non vuole essere un modo per curare lo spirito; tuttavia, come rivelazione del mondo, può anche, cammin facendo, trasformarci nel profondo. La letteratura ha un ruolo vitale da giocare, ma può ricoprirlo solo se viene presa nell'accezione ampia e pregnante che è prevalsa in Europa fino alla fine del XIX secolo e che oggi è stata messa da parte, mentre sta trionfando una concezione assurdamente ristretta. Il lettore comune, continuando a cercare nelle opere che legge come dare un senso alla propria vita, ha ragione rispetto a insegnanti, critici e scrittori quando gli dicono che la letteratura parla solo di sé, o che insegna solo a 
disperare. Se non avesse ragione, la lettura sarebbe condannata a scomparire nel giro di breve tempo (Todorov, 2008, p. 66).

I racconti costituzionali non sono dunque dei discorsi autoreferenziali, ma dei ponti tra le norme costituzionali, gli organi politici e le narrazioni degli attori sociali. I racconti costituzionali hanno a che fare con la realtà costituzionale, essi la descrivono, la invocano, le conferiscono senso e significato.

In questo senso Robert Cover ha offerto un'interpretazione del ruolo della giurisprudenza costituzionale statunitense orientata dall'approccio "diritto e letteratura". Nel suo famoso Nomos e Narrazione, Cover ha indicato nella narratività il ponte che permette di riunire la descrittività con la prescrittività. Mentre il formalismo giuridico irrigidisce la dicotomia tra ciò che è (la realtà fenomenica disordinata) e ciò che dovrebbe essere (le norme giuridiche riunite in un ordinamento), l'approccio "diritto e letteratura" aggiunge una dimensione narrativa, raccontando ciò che è stato ma non è più, ciò che qui non è ma altrove accade, e ciò che potrebbe essere. Secondo Cover si possono distinguere due tipi ideali di normatività: il modello paideico ed il modello imperiale. Il primo

evoca (1) un corpo comune di precetti e narrazioni, (2) un mondo condiviso e personale di essere educati all'interno di questo corpus, nonché (3) un senso di direzione o crescita che si forma mentre l'individuo e la sua comunità elaborano le implicazioni del loro diritto (Cover, 2008, p. 29).

Il modello imperiale, invece, tende alla conservazione del mondo:

al suo interno, le norme sono universali e applicate dalle istituzioni. Esse non hanno bisogno di essere insegnate, almeno fintantoché si rivelano efficaci. Il discorso, in questo modello, è basato sull'oggettività, ovvero sopra ciò che rimane esterno al discorso stesso. Gli impegni interpersonali sono deboli, fondati solo su un obbligo minimo di astenersi dalla coercizione e dalla violenza, fatti che renderebbero impossibile sia articolare il discorso in modo oggettivo, sia l'applicazione imparziale e neutrale delle norme (Cover, 2008, p. 30).

Naturalmente, aggiunge, nessun mondo normativo è mai stato creato o conservato interamente nel modo paideico o in quello imperiale: 
Non sto scrivendo di tipi di società; piuttosto, isolo nel discorso le basi coesistenti dei distinti attributi di tutti i mondi normativi. Ogni nomos deve essere paideico nella misura in cui contiene al suo interno le comunanze di significato che rendono possibile una continua attività normativa. Il diritto deve essere carico di significato nel senso che esso permette a coloro che vivono assieme di esprimere se stessi per mezzo e nel rispetto di esso. Un nomos deve contemporaneamente servire da fondamento per un comportamento prevedibile e fornire significato per l'azione che devia rispetto all'ordinario (Cover, 2008, p. 31).

Mentre la normatività imperiale mira a stabilizzare l'assetto istituzionale, la normatività paideica produce nuovi significati normativi nelle relazioni sociali. I movimenti e le comunità producono normatività nelle loro lotte per il riconoscimento di istanze di giustizia, le istituzioni statali (tra cui le corti) scelgono tra le possibilità interpretative loro offerte dai gruppi. La normatività paideica è giusgenerativa, quella imperiale giuspatica:

l'interpretazione ha sempre luogo all'ombra della coercizione. Tenendo conto di questo fatto, dovremmo giungere a riconoscere un ruolo speciale alle corti. Esse almeno le corti dello Stato, s'intende - sono caratteristicamente "giuspatiche" (jurispathic). Va rilevato, per la sua importanza, il fatto che nei miti e nella storia, l'origine e la giustificazione di un tribunale vengono raramente intese come un bisogno derivato dalla carenza di diritto. Piuttosto la necessità di istituire un tribunale viene interpretata come bisogno di sopprimere parte del diritto, di scegliere fra due o più leggi, oppure imporre una gerarchia fra diritti. È la molteplicità delle leggi, ossia la fecondità del principio giusgenerativo, la causa del problema di cui le Corti e lo Stato vorrebbero costituire la soluzione (Cover, 2008, p. 60).

Nella visione di Cover, i giudici non creano diritto; al contrario essi amministrano la violenza statale sacrificando possibilità interpretative. Seconda questa concezione è la normatività paideica che produce costantemente un sovrappiù di diritto, che la normatività imperiale cerca di ridurre ed ordinare. Ciò presuppone un pluralismo radicale, l'impossibilità di ridurre ad un unità la molteplicità dei mondi normativi delle comunità umane.

È importante sottolineare la natura anarchica di questa visione, "dove per anarchia si deve intendere l'assenza di dominatori, non l'assenza di diritto" (Cover, 2008, p. 97). 
I Codici che legano il sistema normativo alle nostre costruzioni sociali della realtà e alle nostre visioni di ciò che il mondo potrebbe divenire hanno una natura narrativa. La stessa imposizione di una forza normativa su uno stato di cose, reale o immaginario che sia, consiste nell'atto di creare una narrazione. I vari generi della narrazione - storia, romanzo, tragedia, commedia - sono simili nella loro condizione di racconto di uno stato di cose investito da un campo di forza normativo. Vivere in un mondo giuridico richiede che non si conoscano solo i suoi precetti, ma anche le connessioni fra questi ed altri possibili e plausibili stati di cose. Esso richiede che si integri non solo l'essere e il dover essere, ma più precisamente l'essere, il dover essere e il "potrebbe essere". La narrazione è il materiale che integra fra loro questi domini. Le narrazioni sono modelli attraverso i quali studiamo ed esperiamo le trasformazioni che si verificano quando un dato stato di cose viene fatto passare attraverso il campo di forza di un altrettanto semplificato insieme di norme (Cover, 2008, p. 24).

\section{PLURALISMO CULTURALE} NEL DIRITTO E NELLA LETTERATURA

"Il luogo di nascita del romanzo è l'individuo nel suo isolamento"; così Walter Benjamin (2011, p. 19) descriveva la solitudine della genesi del romanzo. A differenza dell'opera d'arte letteraria la costituzione nasce, vive, si trasforma, talvolta muore in una condizione di pluralità; la sua vitalità non può mai essere consegnata nelle mani di un solo costituente, legislatore, giudice $\mathrm{o}$ commentatore accademico. La narratività costituzionale presuppone una pluralità di visioni, di interpretazioni e di lotte per il riconoscimento di istanze sociali.

Dal mio punto di vista, dalla prospettiva di chi studia il diritto costituzionale comparato, l'approccio di "diritto e letteratura" consiste nel riportare lo studio del diritto all'interno di una koinè, di una comunità interpretativa aperta alle differenze dei contesti culturali. Studiando la letteratura l'interprete affina la sua sensibilità nell'interpretazione della realtà, della vita, degli aspetti particolari dell'esistenza, dei valori coinvolti nelle vicende umane; studiando il diritto egli impara il momento drammatico e liberatorio della scelta, tanto delle norme da applicare e dei precedenti cui fare riferimento, quanto degli argomenti da impiegare per persuadere un uditorio. Proprio la condizione giuridica post-moderna, caratterizzata dalla ridondanza cibernetica dei sistemi normativi e dalla 
sovrabbondanza di informazioni giuridiche, è il terreno ideale per coltivare l'abbraccio fecondo tra diritto e letteratura.

Lo studio culturale del diritto si oppone alla pretesa idiotica del formalismo di comprendere il diritto a partire dallo studio del solo diritto. Come l'idiota pretende di capirsi solo a partire dall'esame di se stesso, così il formalismo ha dis-educato intere generazioni di giuristi a studiare il diritto isolandolo dagli altri mondi di vita e contesti sociali in cui il fenomeno giuridico è inserito. Come disse il giurista americano Grant Gilmore, "in hell there is nothing but law"!

Lo studio culturale del diritto si avvale del contributo della letteratura per interpretare i testi classici: le costituzioni, le grandi decisioni dei tribunali, le opere accademiche di ampia diffusione, i documenti politici di rilevanza costituzionale. Come ha rilevato il grande giurista tedesco Peter Häberle (1998), lo studio culturale del diritto si fonda sul pensiero delle possibilità (Möglichkeitsdenken), sulle facoltà immaginative, su ciò che potrebbe essere, sull'uso del congiuntivo e del condizionale. Solo lo studio culturale del diritto può infatti alimentare l'apertura delle società costituzionali contemporanee, apertura al pluralismo normativo, assiologico e sociale. La vita del diritto oscilla tra le esigenze di chiusura del nomos imperiale e le esigenze di apertura del nomos paideico, e il contributo principale degli studi di "diritto e letteratura" consiste proprio nella costante ri-apertura di discorsi, possibilità e mondi di vita, che le esigenze istituzionali e il discorso del potere sempre tentano di richiudere.

Lo studio del linguaggio letterario e di quello giuridico non sono discipline per l'affinamento di tecniche oratorie, di abilità argomentative che possono essere indifferentemente utilizzate per perseguire i fini più disparati; in particolare l'analisi letteraria dei testi giuridici mostra come dentro i concetti giuridici, le categorie dottrinali, le finzioni e le metafore siano riposti principi e valori che sono il frutto del movimento storico. La finzione metodologica della società chiusa aveva permesso alle scienze positive del secolo XIX di considerare il linguaggio come uno strumento manipolabile dal soggetto, ed il significato oggettivo dei testi come il risultato della purificazione dell'arbitrarietà soggettiva dell'interprete. Questo schema è crollato con la crisi della modernità, verificatisi in particolare all'inizio del XX secolo con l'ascesa del Darwinismo, delle 
geometrie non euclidee, della musica dodecafonica, dell'espressionismo e del surrealismo:

\begin{abstract}
questa crisi è infatti inevitabile a partire dal momento in cui si scopre - o si riscopre - la natura simbolica del linguaggio, o, se si preferisce, la natura linguistica del simbolo. È quanto accade oggi, sotto l'azione combinata della psicoanalisi e dello strutturalismo. La società classico-borghese ha visto a lungo nella parola uno strumento o una decorazione; ora noi vi vediamo un segno e una verità. Tutto ciò che è toccato dal linguaggio è dunque, in un certo modo, rimesso in causa: la filosofia, le scienze umane, la letteratura (Barthes 2002, p. 43).
\end{abstract}

Possiamo aggiungere: il diritto.

\title{
RIFERIMENTI BIBLIOGRAFICI
}

AMSTERDAM, Anthony G; BRUNER, Jerome. Minding the Law. Cambridge (MA): Harvard University Press, 2000.

BARTHES, Roland. Critica e verità. Torino: Eiunaudi, 2002.

BARTHES, Roland. The Semiotic Challenge, New York: Hill \& Wang Publisher, 1988.

BENJAMIN, Walter. Il narratore. Considerazioni sull'opera di Nikolaj Leskov. Torino: Einaudi, 2011.

BRUNER, Jerome. La mente a più dimensioni. Roma-Bari: Laterza, 2005.

BRUNER, Jerome. La fabbrica delle storie. Diritto, letteratura, vita. Roma-Bari: Laterza, 2002.

COVER, R. The Supreme Court, 1982 Term. Foreword: "Nomos and Narrative”. Harvard Law Review, v. 97, v. 1, pp. 4-68, nov. 1983.

COVER, R. Nomos e narrazione. Una concezione ebraica del diritto. Torino: Giappichelli, 2008.

DE NITTO, Achille. Introduzione: Consuetudine con la libertà. In: RUPERTO, Cesare. La costituzione in mezzo a noi. Milano: Giuffrè, 2005.

DI DONATO, Flora. La costruzione giudiziaria del fatto. Il ruolo della narrazione nel "processo". Milano: Franco Angeli, 2008.

DWORKIN, Ronald. Law's Empire. Cambridge (MA): Harvard University Press, 1986.

HÄBERLE, Peter. Verfassungslehre als Kulturwissenschaft. Berlin: Duncker \& Humblot, 1998.

NUSSBAUM, Martha. Poetic Justice. The Literary Imagination and Public Life. Boston: Beacon Press,1995.

ORESTANO, Riccardo. Introduzione allo studio del diritto romano. Bologna: Il Mulino, 1987.

TODOROV, Tzvetan. La letteratura in pericolo. Milano: Garzanti, 2008. 
VESPAZIANI, Alberto. Costituzione, comparazione, narrazione. Torino: Giappichelli, 2012.

Lingua originale: Italiano

Ricevuto: 20/02/15

Accettato: 21/04/15 